\title{
Ghost Persistent Scatterers Related to Multiple Signal Reflections
}

\author{
Stefan Auer, Member, IEEE, Stefan Gernhardt, Member, IEEE and Richard Bamler, Fellow, IEEE
}

\begin{abstract}
Persistent scatterer interferometry using stacks of very high resolution SAR data reveals that single or even patterns of scatterers representing building structures may wrongly be localized below the ground level. In this paper, a case study on a test building model is presented using 3D SAR simulation methods in order to explain the underlying localization problem. The case study indicates that ghost persistent scatterers are likely to be related to reflection levels higher than 3. Moreover, the temporal stability of the amplitude of fivefold bounce signals is confirmed for a SAR data stack.
\end{abstract}

\section{Copyright (c)2011 IEEE}

\section{Reprinted from:}

Auer, S.; Gernhardt, S.; Bamler, R., "Ghost Persistent Scatterers Related to Multiple Signal Reflections", IEEE Geoscience and Remote Sensing Letters, vol. 8, no. 5, pp. 919-923, Sept. 2011

Index Terms

3D SAR simulation, persistent scatterer interferometry, multiple signal reflections, TerraSAR-X

\section{INTRODUCTION}

Persistent scatterer interferometry (PSI) [1], [2] based on very high resolution (VHR) SAR data enables the estimation of deformation signals for single objects of interest [3]. The physical origin of long-time coherent SAR image pixels exploited by PSI is not understood to all ends. However, the relation between physical scatterers - e.g. dihedrals, trihrals, or ghost corners [4] - and salient SAR image signatures has to be known in order to understand which object parts are monitored over time.

2D simulators based on ray tracing methods can be used to simulate the appearance of objects on SAR images and to provide test data, e.g. for object detection or object classification [5], [6]. RaySAR, a SAR simulator (developed

S. Auer and S. Gernhardt are with the Chair of Remote Sensing Technology, Technische Universität München (TUM), Germany, e-mail: Stefan.Auer@bv.tum.de, Stefan.Gernhardt@bv.tum.de

R. Bamler is with the Remote Sensing Technology Institute (IMF), German Aerospace Center (DLR), Oberpfaffenhofen, Germany, and with Remote Sensing Technology, Technische Universität München (TUM), Germany, e-mail: Richard.Bamler@dlr.de 
by one of the authors) based on ray tracing methods provides signal contributions in three dimensions including the elevation direction [7]. Thus, the 3D position of simulated signatures can be compared to geocoded persistent scatterers (PSs) localized by PSI. In this regard, PSs related to multiple reflections of radar signals are of special interest.

The work presented in this paper is motivated by practical experiences. Exploiting different VHR spotlight TerraSAR-X data stacks of urban areas by PSI reveals PSs which are wrongly localized below the ground level. However, these mirror images of scatterers, referred to as Ghost-PSs throughout the paper, are not physically present. The real origins of the signals are situated at structures above the ground. While single Ghost-PSs seem to be common for urban areas and are often wrongly interpreted as outliers, patterns of Ghost-PSs are found only for some buildings. A similar effect is reported for bridges over water and is related to multiple reflections of radar signals [8]. In this paper, the results of a case study using SAR simulation methods are presented in order to explain the limitation of the PSI system when localizing PSs related to multiple reflections.

For an urban building in Berlin, represented by a 3D model considering basic facade details, the 3D distribution of signatures is simulated by means of RaySAR. Afterward, the signature positions can be compared to the 3D distribution of PSs localized by PSI. The comparison indicates that Ghost-PSs correspond to radar signals of reflection level 5 whose signal paths are not recovered by PSI. Moreover, it is confirmed that fivefold bounce signals are visible on the SAR images of the data stack.
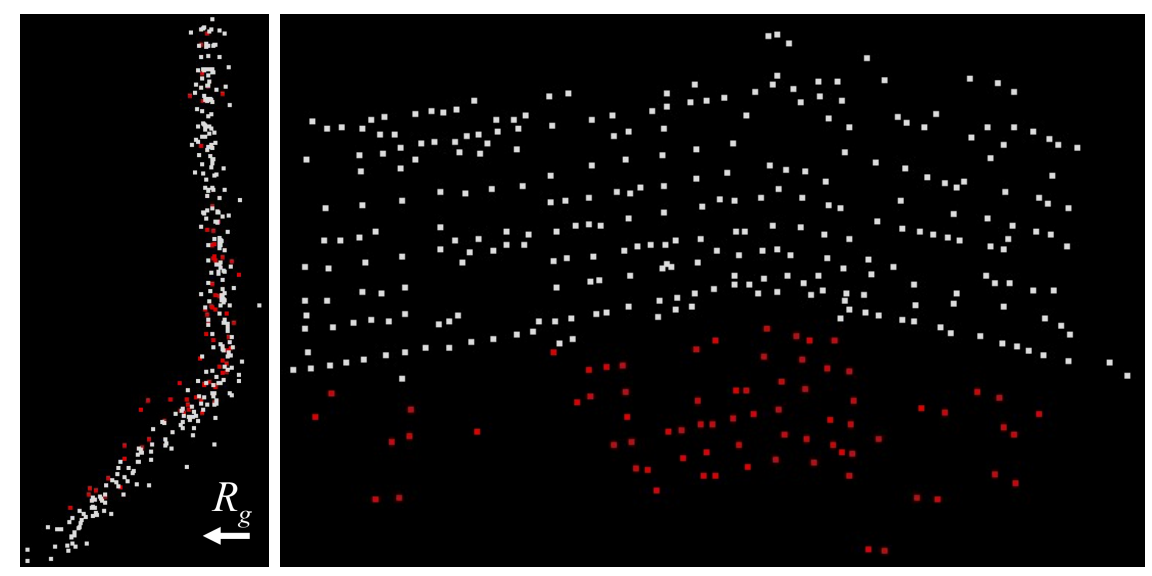

Fig. 1: Geocoded PSs in world coordinates for a building in the center area of Berlin, Germany. Left: perspective top view (vector $R_{g}$ : approximate ground range component of SAR sensor's line-of-sight). Right: perspective side view. Points above and below the ground level are colored in white and red, respectively.

\section{LOCALIZATION OF PSS}

The analysis of the PS positions is motivated by results from PSI processing, using different VHR TerraSAR-X data stacks of the city center area of Berlin. The resolution of the spotlight data is $0.6 \mathrm{~m}$ in range and $1.1 \mathrm{~m}$ in azimuth [9]. Besides information about the deformation of objects, PSI provides the height of each PS relative to 
a reference DEM, i.e. PSs are localized in 3D. Geocoding of the PSs reveals an interesting effect that, for a high number of buildings, PSs may be found beneath the earth surface. Some buildings are even characterized by patterns of Ghost-PSs. An example is given in Figure 1 showing PSs pertinent to a single building of Berlin. The top view onto the distribution of PSs reveals that the majority of PSs are related to two facades and are located in vertical planes. Likely, the deviation from the vertical plane mainly depends on the limited accuracy of the localization in elevation due to the narrow orbital tube of TerraSAR-X. As the average height of the ground level can be estimated from the DEM used for PSI processing, PSs above and below the ground level can be separated (colored in white and red, respectively). On the right part of Figure 1, the spatial distribution of PSs in height is shown from a side view. As SAR sensors in X-Band like TerraSAR-X do not enable to monitor objects below the earth surface, the Ghost-PSs are related to the limited localization capability of PSI.

\section{CASE STUDY BASED ON SAR SIMULATION}

\section{A. Modeling and Sampling}

For SAR simulation, a 3D model of the building is required. As shown in [4], basic facade details such as windows have to be geometrically represented in the model in order to enable a reliable simulation of the appearance of buildings in SAR images. Unfortunately, no such model is available for the building of interest. As a compromise, an approximate geometrical description of the building is accepted. A closer look on facade details is seen in Figure 2. Columns of windows of low depth are interrupted by large window structures of large depth. The front of both window types is composed by glass connected by metallic stripes. Two facades linked by an angle of approximately $130^{\circ}$ describe the basic shape of the building. The ground in front of the building is composed by pavement and two streets made of asphalt.

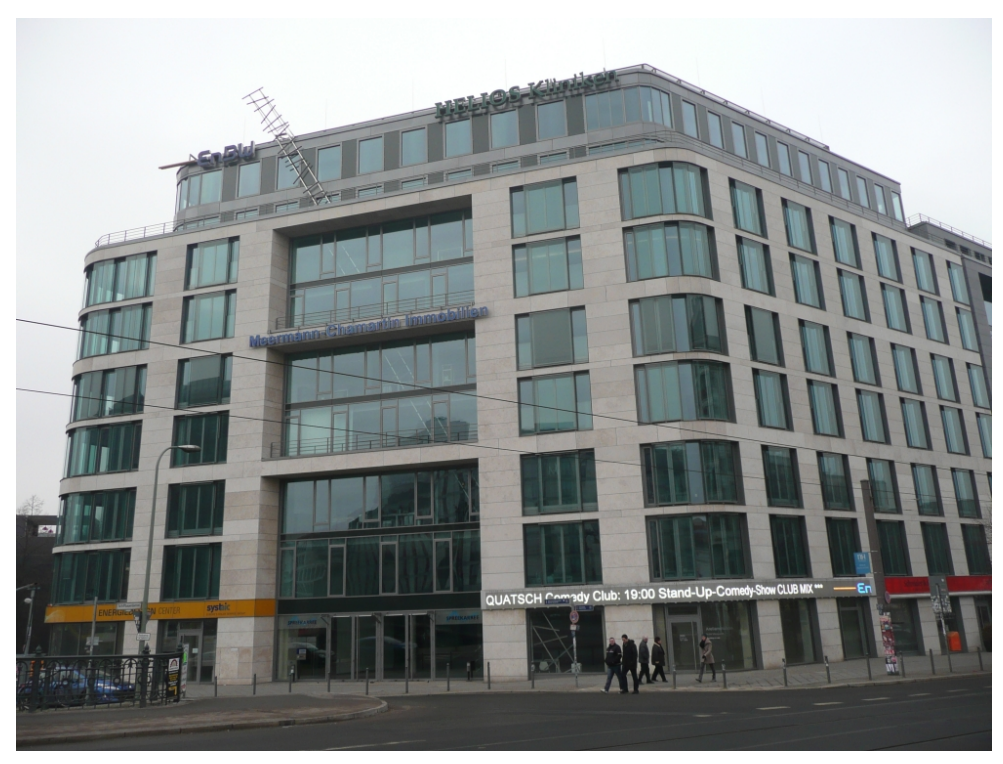

Fig. 2: Perspective view on building of interest. 
Figure 3 shows the 3D model used for SAR simulation which roughly approximates the geometric parameters of the building e.g. building height/length and window dimensions. In contrast, with exception of windows at the curved building corners, the number of windows equals reality. Inserting the windows into the main body of the building is realized by means of Constructive Solid Geometry [10]. The ground beneath the building is represented by a flat plane. Two surface types are defined for considering the reflection of radar signals:

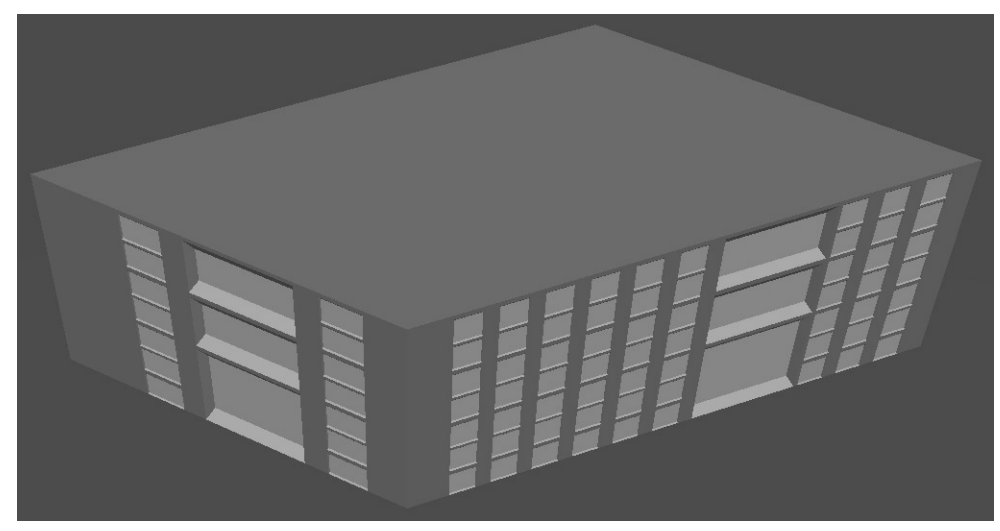

Fig. 3: 3D building model used for SAR simulation.
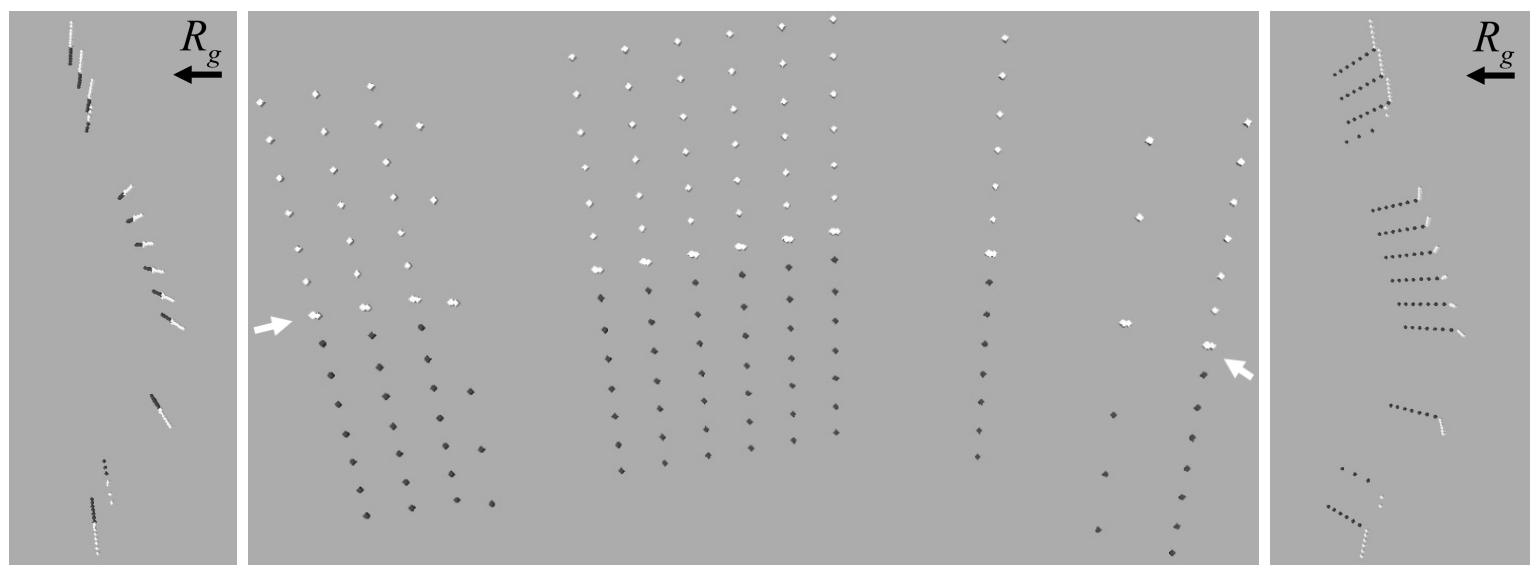

Fig. 4: 3D positions of simulated signatures in world coordinates of the building model (represented by cubes having a sidelength of $20 \mathrm{~cm}$; white: triple bounce; black: fivefold bounce). Left: perspective top view (vector $R_{g}$ : approximate ground range component of SAR sensor's line-of-sight). Center: perspective side view (ground level indicated by arrows). Gaps within the point pattern are related to larger windows. Right: tilt of Ghost-PS pattern for a ground descent of approximately $5^{\circ}$ along both facades (maximum building height at intersection line of both facades).

- building surfaces are assumed to be of metallic material (high reflectivity, strong specular reflection, weak diffuse reflection) 
- the ground surrounding the building is assumed to be dry (medium reflectivity, medium specular reflection, weak diffuse reflection)

As the roughness of surfaces is assumed to be low, the simulation of diffuse reflections is adapted to the Small Perturbation Method (SPM) [11], i.e. relevant diffuse signals are concentrated around the specular direction. The imaging geometry is adapted to the master of the TerraSAR-X data stack (angle of incidence: $36^{\circ}$; approximated aspect angle with respect to the building). Eventually, after the modeling step, signal contributions are simulated by RaySAR taking 4 min $15 \mathrm{sec}$ on a standard PC (2 GHz Dual Core, 4 GB RAM). RaySAR represents an ideal SAR system, i.e. signal contributions are simulated with infinite resolution in azimuth, range, and elevation [7].

\section{B. Distribution of simulated signatures}

Given the sensor position and the imaging geometry with respect to the building, the position of the simulated signatures - in azimuth, range, and elevation - can be transformed into the world coordinate system of the simulated building model. Thereafter, the signatures are exported to a CAD model file where they are represented by cubes. For the case study, the result is shown in Figure 4. In this regard, the focus is on dominant signal contributions, i.e. signal contributions of weak amplitude are discarded. Only triple and fivefold bounce signals, colored in white and black, respectively, are distinguishable.

As for the geocoded PSs localized by PSI, the simulated signatures are grouped in vertical patterns. Triple bounce signatures are found above the ground and on ground level. In contrast, fivefold bounce signatures are found below the ground level. Compared to the pattern of PSs in Figure 1, the simulated pattern shows more regularity. This is due to the infinite resolution of the virtual SAR system while the resolution of the real SAR system is limited, especially in the elevation direction. Obviously, the simulated signatures of bounce level 3 are less in numbers than the detected PSs. This meets our expectations as the simulated 3D building model is only a rough approximation of the real building. For instance, metallic structures within the windows and structures on the building roof may cause further prominent point signatures but are not considered by the 3D model. In contrast, the number of simulated signatures below the ground level is higher than the number of Ghost-PSs in the real data. Different factors may be responsible for the loss of PSs which are discussed in Section III-D.

Nonetheless, the case study confirms that the occurrence of Ghost-PSs is deterministic. An explanation and discussion of this effect is given in the following.

\section{Occurrence of Ghost-PSs}

Imagine a basic building model containing two windows, shown in in Figure 5 whose facade is parallel to the SAR sensor's line-of-flight. As the model is located in the far field with respect to the SAR antenna, the signal wavefront is assumed to be locally flat.

Strong double bounce is expected due to signal interaction with both the ground and the building wall. Further specular double reflections of radar signals occur at two dihedrals formed by the windows. After geocoding, the interferometric SAR system localizes the corresponding signal origin on ground level (see big black cross in the 


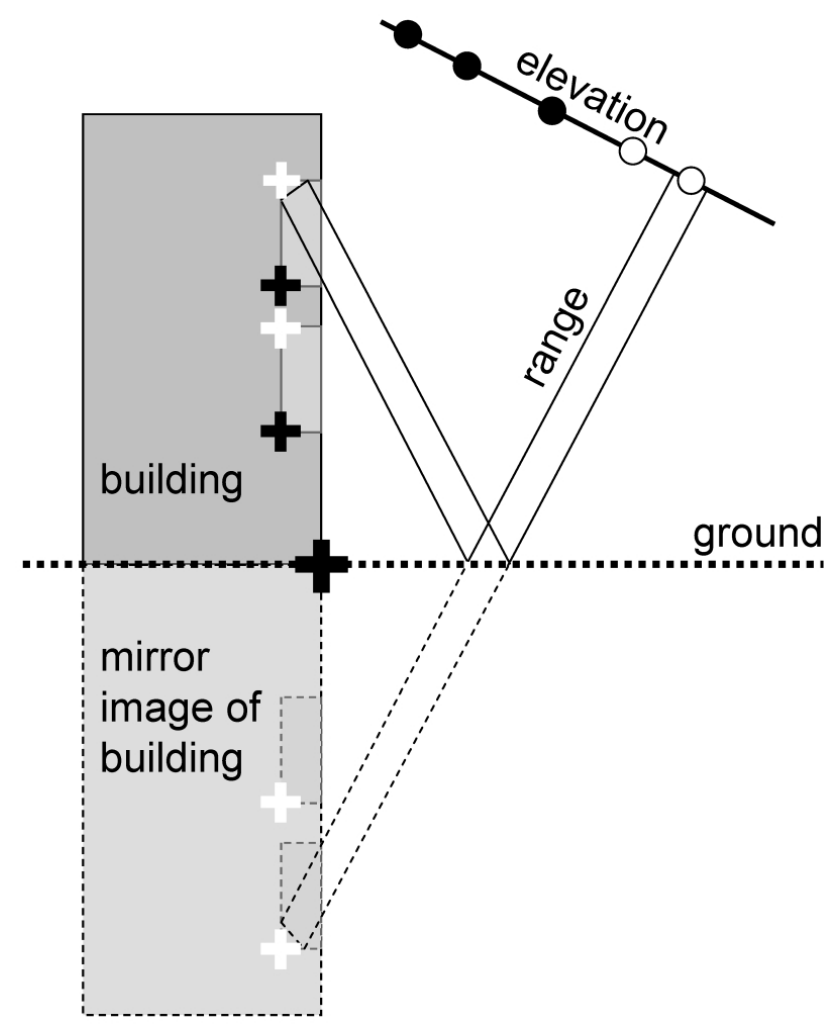

Fig. 5: Mirror effect for facade with two windows facing the SAR sensor, displayed in the range-elevation plane. Black crosses: origin of double reflections. Upper white crosses: origin of fourfold bounce signals. Bottom white crosses: mirror position of fourfold bounce signals localized by PSI. Circles mark the pertinent elevation positions.

center of Figure 5) and at both windows (smaller dark crosses). The elevation positions of double bounce signatures are marked by black dots.

In addition, fourfold reflections occur due to signal interaction with the ground and windows (signal path: ground $\rightarrow$ window front $\rightarrow$ window top $\rightarrow$ ground and vice versa; see Figure 5). In order to enable this reflection effect, the window front interacting with the radar signal has to be partly covered with other material than glass. SAR systems are not able to resolve this scattering mechanisms as the signal path between the first and last bounce can not be reconstructed. The elevation positions of the signal origins are found in between the elevation positions of the first and last bounce (marked by white dots), respectively [7]. Moreover, the range coordinate is given by half of the distance the radar signal travelled. Hence, after geocoding, the fourfold bounce signatures are localized below the ground level (bottom white crosses in Figure 5). The positions of the real signal origins (upper white crosses) can be found by mirroring the ghost scatterers at the ground level.

The case of Figure 5 is a special one. In most cases, like for our building of interest, the facades are not aligned parallel to the azimuth direction. Hence, instead of double reflections, triple reflections occur locally at window corners (signal path: window bottom $\rightarrow$ window side $\rightarrow$ window front and vice versa). Multiple reflections including 
the ground are of reflection level 5 (signal path: ground $\rightarrow$ window front $\rightarrow$ window top $\rightarrow$ window side $\rightarrow$ ground and vice versa). In contrast to signal reflections shown in Figure 5, the triple and fivefold bounces of the radar signals are not limited to the range-elevation plane but also cover the azimuth direction.

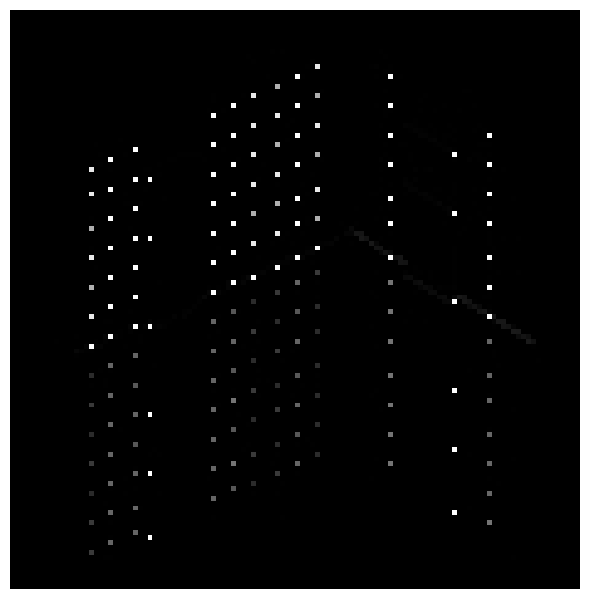

(a) Simulated reflectivity map

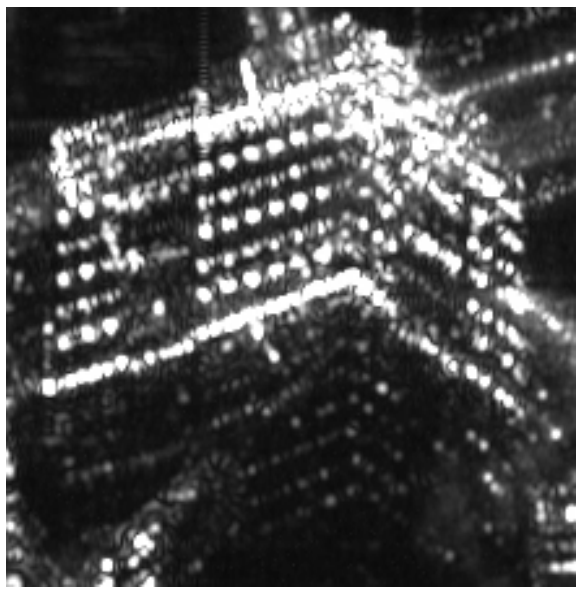

(c) SAR temporal average image

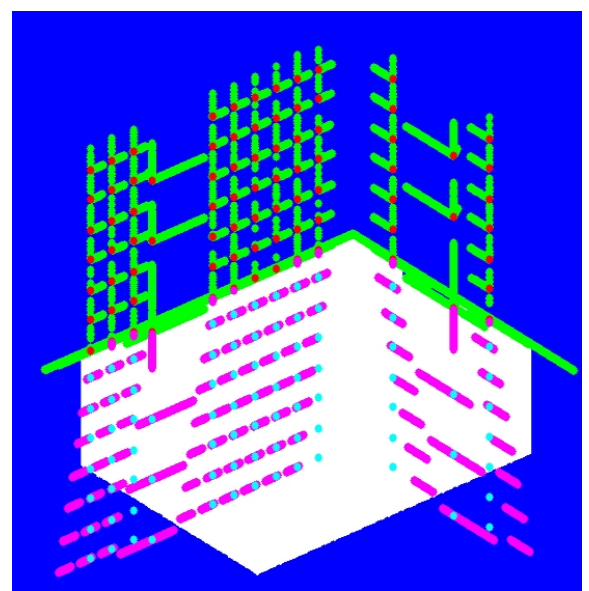

(b) Distribution of signal responses; blue: single bounce; green: double bounce; red: triple bounce; magenta: fourfold bounce; cyan: fivefold bounce; white: shadow

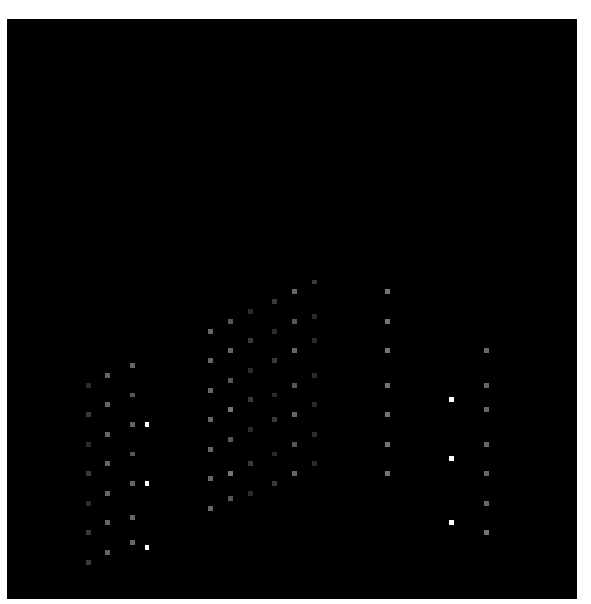

(d) Simulated fivefold bounce

Fig. 6: Simulation vs. SAR temporal average image. SAR simulation reveals that triple and fivefold bounce signatures are prominent while double and fourfold reflections are negligible. Fivefold bounce signatures can be distinguished on the SAR image. Linear signatures likely correspond to a combination of double and triple reflections. Range: top-down. 


\section{Discussion}

The 3D geometry shown in Figure 5 is often present in urban areas. We believe that PS patterns below the ground level is a consequence of the imaging geometry (and of the implicit assumption of the 1st Born approximation), rather than outliers. For the center area of Berlin, regular patterns of PSs comparable to the example shown in Figure 1 are found only for some buildings. In contrast, single PS or small groups of irregularly distributed PSs below the ground level are found for a high number of buildings. From practical experience, Ghost-PSs are likely for

- isolated buildings on flat ground covered by asphalt or concrete. Vegetation or objects covering the ground next to the building attenuate specular reflections in direction to the windows. Any deviation of the ground from a flat horizontal plane will distort the mirror PS pattern. A linear descent of the ground in front of the facade causes an increase of the signal path ground $\rightarrow$ window depending on the window position with respect to the ground level. Due to the increase of the corresponding range coordinates, the Ghost-PSs are shifted out of the vertical plane. Figure 4 right shows the simulated position of signatures for linear descent along both facades of the building model. An ascent of the ground would cause a shift in the opposite direction.

- facades characterized by window patterns, as the number of PSs is related to the number of windows. Depending on the incidence angle of the radar signal, the window depth is crucial for the signal reflection at window corners. The vertical extension of the PS pattern depends on the vertical distribution of windows.

- window frames made of metallic material. For materials of low permittivity, the signal power is negligible for reflection levels larger than 3 .

The characteristics of PSs localized beneath the ground level are of interest for PSI processing. Relative deformations between buildings and the ground, e.g. tilting or rotation, is included twice as the path between the ground and the window is traveled twice by the radar signal.

\section{E. Simulation vs. TerraSAR-X image}

As PSs are selected due to long-time coherent amplitude values, point signatures of bounce level 5 should be visible for the building of interest on the TerraSAR-X images of the data stack. In order to support the visual interpretation, a reflectivity map is simulated using RaySAR. To this end, discrete signal contributions provided by the ray tracer are imposed with a regular grid and are summed coherently within each grid element. The pixel size is adapted to the VHR spotlight TerraSAR-X data, i.e. $0.433 \mathrm{~m} \mathrm{x} 0.383 \mathrm{~m}$ in azimuth and ground range. A high clipping level on the intensity is chosen in order to concentrate on prominent signatures. In Figure 6a, the resulting reflectivity map is shown. The appearance of the building is characterized by two point patterns of high and medium amplitude, respectively, which correspond to reflection levels 3 and 5. The distribution of simulated signal responses seen in Figure $6 \mathrm{~b}$ reveals that single, double, and fourfold reflections are present as well but are too weak to be distinguished on the reflectivity map (with except of two double bounce lines on the right part of Figure 6a). 
In Figure 6c, a SAR image of the urban scene is shown which has been created by averaging 36 VHR spotlight TerraSAR-X images. With the support of the simulated map, the image can be interpreted top-down in range direction. First, triple reflections are found in a layover area. Thereafter, the bottom end of facades are represented by bright lines. These signatures are not distinguishable on the reflectivity map but are confirmed by the simulated distribution of signal responses (see green lines in Figure 6b). Optimizing the surface parameters of the building model or choosing a different clipping level for the reflectivity map may remove this difference. A point pattern of low amplitude follows after the end of the layover area which corresponds to simulated fivefold bounce signals seen in Figure 6d. Hence, even if the 3D model is only a rough approximation of the real building, the simulated maps give a strong hint that fivefold bounce signals are visible on the SAR image.

\section{Conclusions}

The case study presented in this paper indicates that fivefold bounce signatures are distinguishable in VHR SAR data of meter-resolution and are exploited as PSs. Likely, the main factors influencing this reflection phenomenon are the presence of window patterns, flat ground surrounding the building, and appropriate surface materials on both the ground and the building. Due to the implicit acceptance of the 1st Born approximation by the PSI system, PSs corresponding to fivefold bounce are localized in vertical patterns below the ground level.

Simulating a generalized 3D model representing basic facade structures by means of ray tracing methods has proven to be helpful for explaining the geocoding problem. This is of interest, as the availability of very detailed 3D models for non-prominent buildings is not common.

\section{REFERENCES}

[1] A. Ferretti, C. Prati, and F. Rocca, "Permanent scatterers in SAR interferometry," IEEE Transactions on Geoscience and Remote Sensing, vol. 39, no. 1, pp. 8-20, Jan 2001.

[2] B. Kampes, Radar Interferometry - Persistent Scatterer Technique, F. van der Meer, Ed. Dordrecht, The Netherlands: Springer, 2006.

[3] S. Gernhardt, N. Adam, M. Eineder, and R. Bamler, "Potential of very high resolution SAR for persistent scatterer interferometry in urban areas," Annals of GIS, vol. 16, no. 2, pp. 103-111, 2010.

[4] S. Auer, T. Balz, S. Becker, and R. Bamler, "3D SAR simulation of urban areas based on detailed building models," Photogrammetric Engineering \& Remote Sensing, Journal of the American Society for Photogrammetry and Remote Sensing, vol. 76, no. 12, pp. 1373-1384, 2010.

[5] H. J. Mametsa, F. Rouas, A. Berges, and J. Latger, "Imaging radar simulation in realistic environment using shooting and bouncing rays technique," in Proceedings of SPIE Conference, SAR Image Analysis, Modeling and Techniques IV, 2001.

[6] G. Margarit, J. J. Mallorqui, and C. Lopez-Martinez, "GRECOSAR, a SAR simulator for complex targets: Application to urban environments." in Proceedings of IEEE International Geoscience and Remote Sensing Symposium (IGARSS), 2007, pp. 4160-4163.

[7] S. Auer, X. Zhu, S. Hinz, and R. Bamler, "Ray tracing and SAR-tomography for 3D analysis of microwave scattering at man-made objects," in International Archives of Photogrammetry, Remote Sensing and Spatial Information Sciences, vol. 38-3/W4, 2009, pp. 157-162.

[8] U. Soergel, E. Cadario, A. Thiele, and U. Thoennessen, "Feature extraction and visualization of bridges over water from high-resolution InSAR data and one orthophoto," IEEE Journal of Selected Topics in Applied Earth Observations and Remote Sensing, vol. 1, no. 2, pp. 147-153, June 2008.

[9] W. Pitz and D. Miller, "The TerraSAR-X satellite," IEEE Transactions on Geoscience and Remote Sensing, vol. 48, no. 2, pp. 615 -622, feb. 2010 . 
[10] C. M. Hoffmann, Geometric \& Solid Modeling. Morgan Kaufmann Publishers, San Mateo, California, 1989. [Online]. Available: http://www.cs.purdue.edu/homes/cmh/distribution/books/geo.html

[11] L. Tsang and J. Kong, Scattering of Electromagnetic Waves: Theories and Applications, L. Tsang and J. Kong, Eds. John Wiley \& Sons, Inc., New York, 2001. 